\title{
Q Género y Ciencia. A propósito DE LOS "ESTUDIOS DE LA MUJER" EN LAS UNIVERSIDADES
}

\author{
Consuelo Flecha García
}

Universidad de Sevilla

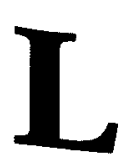

os Estudios de la Mujer, aunque representan un campo del saber relativamente reciente, han demostrado una gran capacidad de desarrollo y de institucionalización en las Universidades de muchos países, incluida España. De su casi inexistencia hace sólo tres décadas, se ha pasado a una situación en la que están presentes y son reconocidos en los ámbitos académicos como un nuevo campo de investigación y de docencia.

En el caso español, no ha sido ajeno a ello la participación de las mujeres en muchos espacios de la vida pública, su presencia en distintas instituciones, su incorporación, como estudiantes y como profesoras, a los niveles educativos superiores, uno de los hechos más significativos de los producidos, por ejemplo, en la Universidad española de los últimos treinta años'. Presencia que, durante muchas décadas, no cambió ni planes, ni programas,

\footnotetext{
' El porcentaje de alumnas universitarias, en primero y segundo ciclo, ha pasado de un $30,3 \%$ en el curso 1972-73, a un 52,5\% en el de 1994-95; en tercer ciclo las estudiantes suponían en $1990-91$, el $34,4 \%$, y en $1994-95$ el $47,49 \%$. La presencia de profesoras ha crecido desde el $15 \%$ que representaban en 1972-73, al 31,5\% del curso 1994-95. Sin embargo, la distribución por carreras y disciplinas, aunque se están produciendo cambios significativos, sigue siendo aún muy desigual (Flecha, 1987; Instituto de la Mujer, 1988; I.N.E., 1991 a 1995).
} 
ni conceptos, ni comportamientos, ni estructuras, puesto que los modos concretos que el proceso histórico había ido consolidando a lo largo de los ocho siglos de existencia de esta institución, se asumían como universales aunque lo fueran sólo masculinos. Las universitarias transitaban un espacio cargado de la tradición de quienes lo habían diseñado, establecido y ocupado con la conciencia de que sólo a ellos pertenecía, y en el que se habían acumulado olvidos, ausencias y prohibiciones. Pero la fuerza de la realidad y de la evidencia ha empezado a remover algunas de estas certezas.

Aunque no sólo por eso, el desarrollo de los Estudios de la Mujer, ampliamente abordados en Estados Unidos y en varios países europeos, se produjo más lentamente en las Universidades españolas. La situación social y política de la realidad nacional en las décadas sesenta y setenta, la ausencia de un movimiento de mujeres que fuera más allá de algunas reivindicaciones políticas en el tema de la igualdad, y la dificultad estructural, económica y social de la Universidad para abrirse a nuevos planteamientos dificultó que estos temas entrasen como objeto de investigación y de docencia en la Academia.

La incorporación se realizó primero en Estados Unidos y en Inglaterra, en el marco de un movimiento feminista que, reclamando la igualdad en los años sesenta, empujó a un grupo de profesoras universitarias a introducir este objetivo en su trabajo docente e investigador a través de las propias disciplinas académicas. Más tarde, apenas hace dos décadas, se hizo lo mismo en algunos países europeos en los que, con distinto ritmo, se fueron implantando e institucionalizando los Estudios de la Mujer en las Universidades. Las experiencias y estrategias de consolidación de todo el conjunto de actividades de docencia, de investigación y de difusión de conocimientos en torno a las mujeres necesitó, sin embargo, en todas ellas de un esfuerzo colectivo y sistemático para que fueran introducidos en los planes de estudios oficiales. La doble función, científica y social de la Universidad, su capacidad para legitimar unos conocimientos y para favorecer su aceptación social, así como la posibilidad de difusión de los mismos mediante la política de publicaciones de que normalmente disponen fueron argumentos que sostuvieron a las iniciadoras en el proceso que tuvieron que recorrer.

La presencia creciente en las Universidades europeas, y algunas de las inquietudes que fueron suscitando, llevó a que en 1984 la Comunidad Europea elaborara un Informe sobre "Donne: ricerca nei dieci paesi della Comunitá Europea" (Donne d'Europa, 1984), a que en 1989 organizara la Primera Conferencia Europea de Estudios de la Mujer bajo el título de "Women's Studies Concept and Reality", y a que en 1994 el Parlamento Europeo se interesara y propusiera medidas para paliar las diferencias en el personal científico dedicado a $I+D$ en Europa (Parlamento Europeo, 1994). Igualmente, desde 1988, a través del Proyecto GRACE, viene promoviendo la formación de un Banco de Datos sobre Estudios e Investigaciones Feministas en Europa, del que ha publicado ya varios informes (Comisión Europea, 1992; Equipe Simone, 1996). 
La evaluación y diagnóstico de toda esta realidad ha llevado a la Unidad para la Igualdad de Oportunidades de las Comunidades Europeas a introducir en el "III Programa de Acción para la Igualdad", medidas relacionadas con los Estudios de las Mujeres, a la elaboración de un Informe sobre estos estudios en el marco de la integración europea (Hanmer, 1994), y a la aprobación de varias Resoluciones relacionadas con la presencia de mujeres en los ámbitos científicos y tecnológicos ${ }^{2}$.

\section{DESARROLLO DE LOS ESTUDIOS DE LA MUJER}

La normalización de los Estudios de la Mujer en el mundo académico -es decir de unos estudios que aspiran a renovar las disciplinas utilizando categorías analíticas y de interpretación que cuestionan y critican una visión de la ciencia objetiva y neutra-, se ha ido produciendo a través del desarrollo de programas de investigación financiados por las Universidades y por otros organismos científicos, con la realización de seminarios y de cursos complementarios, con la incorporación de asignaturas específicas a los planes de estudios, etc. En la actualidad existe una compleja articulación de programas de estudios organizados dentro de la dinámica universitaria, que han incorporado a las mujeres como un elemento central de la docencia y de la investigación; hecho que, además de proporcionar al alumnado una oferta de elección más amplia en el diseño de su propio currículum, ha supuesto un paso imprescindible para garantizar un más amplio desarrollo y difusión de los conocimientos científicos en torno a las mujeres (Nash, 1988, 21. TobeSteen, 1993. Krops, 1997). Hoy, Estados Unidos y otros países cuentan con Licenciaturas, Cursos de Postgrado, Masters y Programas de Doctorado en los que, por su carácter interdisciplinar, colaboran docentes de diferentes áreas científicas y departamentos, dando a los mismos un carácter y una dimensión más abierta y plural, y haciendo posible tanto la formulación de preguntas diferentes como la búsqueda de soluciones a las nuevas necesidades intelectuales planteadas desde la perspectiva de las mujeres.

Acercarse e interrogar a las diferentes disciplinas con nuevos criterios, construir esquemas interpretativos distintos, utilizar nuevos conocimientos que incluyen ya a las mujeres, está ayudando a una mejor comprensión de las experiencias femeninas, al replanteamiento de su identidad y al desarro-

\footnotetext{
${ }^{2}$ En 1994 se aprobó una Resolución "Sobre el personal científico dedicado a la I+D en Europa" que aborda el nivel de presencia de mujeres en la investigación, como consecuencia de los resultados y recomendaciones del "Seminario Internacional sobre Las Mujeres en la Investigación nes cientica y Tecnológica", celebrado en 1993 por el STOA (Programa de evaluación de las opciones científicas y tecnológicas) en el Parlamento Europeo.
} 
llo de una cosmovisión desde una perspectiva del conocimiento que tiene en cuenta a la población femenina. Parece que, así como la educación fue entendida en el siglo XIX por muchas mujeres como un medio fundamental para cambiar la situación y condición que se les había asignado, ahora los Estudios de las Mujeres se están percibiendo como el instrumento que mejor puede contribuir a la transformación del mundo en el que se mueven y de la perspectiva desde la que son consideradas en la sociedad.

En Europa empezó en los años setenta el desarrollo de Cursos en Universidades de diferentes países; en la Universidad de París se impartió en 1973-74 un curso sobre " ¿Tienen historia las mujeres?" (Perrot, 1984) y en la Universidad de Oxford otro sobre “¿Existe una historia de las mujeres?” (Degler, 1975). Fue el comienzo de una actividad docente que enseguida se ampliaría con otras en esos mismos países, para extenderse a continuación al resto de Europa.

En Francia, una larga lista de Jornadas y Congresos celebrados desde 1975, así como de publicaciones de obras y de revistas -Histoires d'Elles, Questions féministes, Clio-, pone de manifiesto el camino recorrido por las Universidades francesas en los últimos veinte años (Anef, 1995, 689-710); período en el que se han creado Centros y Grupos de investigación y de docencia sobre campos específicos o interdisciplinares en diferentes Universidades (Martin, 1993, 24-28).

En 1995 Inglaterra contaba con más de 30 instituciones universitarias que ofrecian programas de Estudios de la Mujer dentro de los planes de formación de sus estudiantes (Bird, 1995), las Universidades de los Países Bajos impartían un total de 260 cursos (Jansen-Alst, 1995), y, en Suecia, todas las Universidades incluían esta modalidad de estudios; en los tres países, después de recorrer un proceso iniciado en los años setenta, y que fue institucionalizándose en la década de los ochenta (Ericsson, 1995). En Alemania también se iniciaron, junto con los movimientos de mujeres, en los años setenta, dentro y fuera de la Universidad; Irlanda empezó a acogerlos en los ochenta, y Portugal, donde se ha formado una Associçao Portugesa de Estudos sobre as Mulheres, cuenta igualmente con estos estudios en varias de sus Universidades (Múller, 1995; Cháthaigh, 1995; Reynolds, 1993).

Grupos de mujeres y profesoras universitarias han hecho posible en Italia un gran desarrollo de los Estudios de la Mujer, aunque haya sido fuera de las Universidades, pues la organización centralizada de la enseñanza superior italiana ha impedido su incorporación a los estudios académicos formales; sin embargo, muchas disciplinas y muchas investigaciones dentro de la Universidad se desarrollan desde esta perspectiva, además de organizar Seminarios, Jornadas y Congresos que favorecen la reflexión y el trabajo (Addis, 1992). Aunque con un peso menor en cuanto a la dedicación y volumen, la misma característica centralizadora ha impedido su presencia formal en las universidades de Grecia. También países como Rusia, que ha crea- 
do dentro de la Academia de Ciencias el "Moscow Center for Gender Studies", Polonia, Hungría, Rumanía, la República Checa, han incorporado en los últimos años estos estudios a sus Universidades y Centros de educación superior (Krops, 1997).

\subsection{SU INCORPORACIÓN A LAS UNIVERSIDADES EN ESPAÑA}

España se ha incorporado ya, y muy activamente, a este movimiento internacional que se ocupa de los Estudios de las Mujeres. La celebración del Año Internacional de la Mujer en 1975, además de propiciar la presentación pública del feminismo, despertó inquietudes que habían permanecido latentes durante varias décadas, entre ellas la de abordar una reflexión que se centrara en la mujer -enseguida, las mujeres- desde nuevas perspectivas. Las formas de convivencia democrática que se preveían en aquellos años animó a replantear el análisis del pasado de la condición femenina asignada, aunque, en realidad, estas inquietudes, estas relaciones de dependencia colectivamente concienciadas, tenían raíces y conexiones más profundas en el tiempo.

Milagros Rivera ha escrito que "no es de hoy ni del siglo XIX. Es una relación que existe desde que las mujeres (o grupos de mujeres) tomaron conciencia colectiva de su subordinación social y escribieron sobre ella" (Rivera, 1996, 43), desde que Christine de Pizán intervino a principios del siglo XV en el debate llamado Querella de las Mujeres, en los albores del Humanismo. En España, a partir del siglo XVIII, desde la palabra de ilustradas como Josefa Amar y Borbón y desde las acciones de las que, en torno a la Junta de Damas de Honor y Mérito, buscaban nuevas posibilidades para las mujeres; desde los cada vez más numerosos escritos, a lo largo del siglo XIX, que irían reflejando tanto una progresiva toma de conciencia de la situación en que se hacía vivir a las mujeres, como las propuestas y medidas que era necesario tomar para cambiarla. Concepción Arenal, Emilia Pardo Bazán, Concepción Gimeno o las iniciativas puestas en marcha en torno a la Asociación para la Enseñanza de la Mujer y a otros grupos de mujeres organizadas en torno a Revistas, Ateneos, Escuelas Normales, Asociaciones Culturales y de Caridad, etc., que veían en la educación el mejor camino de salida, pusieron rostro y argumentos concretos a unas inquietudes y a unos deseos que crecieron significativamente en las décadas previas al cambio de siglo.

Los movimientos que en nuestro país tuvieron más fuerza y protagonismo en el nuevo siglo fueron los que giraban en torno al acceso de las mujeres al trabajo remunerado, a la cultura superior y a la ciencia. El impulso que en este sentido ofrecieron la Junta para la Ampliación de Estudios, la Escuela de Estudios Superiores del Magisterio, las Academias y Residencias Universitarias Teresianas, la Residencia de Señoritas, así como el protagonismo de mujeres como María de Maeztu, Josefa Segovia, Victoria Kent, 
María de Echarri, Carmen Cuesta, María Lejárraga y muchas otras, fueron un nuevo eslabón de esa genealogía que ha llegado hasta la actualidad, cada vez más acompañada de nombres concretos y de realizaciones gratificantes.

Décadas más tarde, en una fecha y en un contexto con connotaciones muy especiales para el tema que nos ocupa, Ángeles Galino afirmó que "guste o no, el hecho está ahí: nuestro siglo ha visto modificarse profundamente el estatuto social de la mujer que, en algunos sectores, ha contado con posibilidades de formarse semejantes a las del varón, y ha accedido así a puestos de trabajo y responsabilidad tradicionalmente vedados para ella" (Galino, 1972, 110); constatación con la que esta Catedrática universitaria, la primera en España, demostraba la permanencia de una conciencia y de una voluntad que se resistía a mantenerse en la subordinación, aunque durante varias décadas el mensaje oficial transmitido se hubiera movido en esas coordenadas.

Cuando, pasados unos años, se quiso incorporar el discurso de las mujeres a la vida social, primero, y a la vida académica, después, no se contaba con estructuras de apoyo institucionales, y las mujeres representaban una exigua minoría entre el profesorado universitario. Quizás por eso, los primeros pasos se debieron más a iniciativas individuales, o incluso a grupos de mujeres no siempre vinculadas directamente con la Universidad, que tenían que abordar estos estudios en régimen de "doble jornada científica", por la falta de legitimidad y de reconocimiento de este tipo de actividades e investigaciones en el ambiente universitario de aquellos años (Moreno, 1977, 61-67).

La tarea realizada por esas pioneras, los contactos y relaciones con otras mujeres que se fueron uniendo en los mismos objetivos, y la misma apertura de los horizontes del conocimiento propiciaron, ya al final de esa misma década, la creación de los primeros Seminarios de Estudios de la Mujer en las Universidades Autónomas de Madrid y de Barcelona, a los que seguirían otros en los años siguientes. Apoyados más en la decisión y voluntarismo de profesoras y alumnas que, todavía, en el respaldo y reconocimiento institucional, se pusieron a trabajar y se dieron a conocer con la organización de Jornadas y de Seminarios, con Publicaciones y con Proyectos de Investigación que alentaron y contribuyeron a la introducción de una perspectiva diferente en la docencia por parte de quienes participaban en esas actividades. Se puede considerar la década siguiente, la de los años ochenta, como la de la consolidación de una voluntad y de unas acciones que han demostrado tenían una significativa capacidad de desarrollo (Ballarín, Gallego y Martínez, 1995).

En apenas veinte años se ha acumulado una gran producción de realizaciones y de conocimientos que van cubriendo los infinitos huecos de la ciencia y de las estructuras en las que no estaban las mujeres, que van sacando a la luz su existencia, que las incorporan a las disciplinas académicas y a cualquier espacio científico y social. Se trataba, en un primer momento, de hacer visibles a las mujeres en los saberes, de reconocerles espacios más allá de lo 
doméstico, y se trabajó con inteligencia y con constancia para acelerar un proceso que parecía avanzar demasiado lentamente.

La demanda social de este tipo de temas y de planteamientos ha ido incrementándose, y sus efectos se aprecian en muy diversas manifestaciones: en la cada vez más rápida multiplicación de los programas de estudios de la mujer en las universidades y en otros centros de enseñanza, en los programas sindicales de formación, en los de asociaciones culturales de mujeres, vecinales, etc., en los nuevos proyectos curriculares de formación inicial y permanente del profesorado, en la organización de bibliotecas y de centros de documentación específicos, en la creación de colecciones dentro de los Servicios de Publicaciones de varias Universidades y de Editoriales comerciales, en los congresos sobre estudios de las mujeres, en los movimientos de salud, de tecnología, de violencia, de autoayuda para mujeres. Toda una variedad de realizaciones que atestigua el desarrollo y atención que están mereciendo por parte de muchas personas y organismos.

\subsection{LOGROS Y PROSPECTIVAS}

La panorámica que nos ofrece la experiencia acumulada en apenas tres décadas permite destacar algunas cuestiones que ponen de manifiesto el camino recorrido, los huecos que van apareciendo y las tareas que quedan por cubrir. Se puede afirmar que se ha experimentado una evolución y una madurez progresiva en las investigaciones y en la reflexión sobre teoría feminista, sobre la aplicación del género como categoría de análisis, y se han abierto perspectivas que pueden ser aplicadas a otros campos del saber.

La consolidación de Cursos, de Programas y de Investigaciones en el ámbito universitario va logrando que estos estudios sean aceptados y reconocidos en lo que pueden aportar, como nuevo campo de conocimiento, dentro de las diferentes disciplinas. La aceptación del alumnado, respondiendo a las convocatorias que bajo estas denominaciones se hacen, y el interés con que participan en los cursos y actividades son la mejor expresión de los cambios producidos y de las transformaciones que aún aguardan. Lo mismo sucede con una parte del profesorado, progresivamente más dispuesto a introducir los análisis de género o desde las mujeres en la presentación y desarrollo de sus disciplinas.

El tipo de cuestiones que se analizan y estudian, el compromiso a que llevan con la propia realidad y con la del medio en el que se vive y "la búsqueda de rigor científico en las investigaciones (...) ha puesto de manifiesto la importancia del tema y la imposibilidad de soslayarlo" (Gómez, 1995, 17), bios sulmente en aquellas áreas científicas que estén experimentado cambios sustantivos o que presenten un cierto dinamismo. 
Pero sólo se ha iniciado el camino, aunque haberlo logrado parezca un objetivo ya suficientemente amplio. Queda mucho hasta que sea posible una ciencia, una cultura y una educación no sesgadas en razón del sexo, como tampoco tienen que serlo por otros indicadores sociales como la raza, la clase social, la procedencia geográfica, cultural o religiosa.

\section{GÉNERO Y CIENCIA}

La labor realizada en estos años ha producido reflexión, análisis y un nuevo modo de acercarse a los paradigmas científicos que guiaban el camino hacia el conocimiento. Ciencia y feminismo, búsqueda del saber y contexto en el que éste se genera, tradición científica y nueva ciencia son cuestiones que han llevado a abrir un diálogo, un debate, una confrontación, sobre el verdadero carácter de los saberes históricamente acumulados y sobre la necesidad de una redefinición de los mismos y de las visiones del mundo que nos han transmitido. Los estudios sociales de la ciencia, que la sitúan en su contexto social y político, la utilización en la misma de nuevas técnicas metodológicas, la relevancia que está adquiriendo la experiencia personal y la subjetividad en la reflexión teórica, y el análisis crítico de muchos de los supuestos fundamentales que han prevalecido en las diferentes ciencias no son ajenos a los cambios que precisamente todo ello está provocando.

Igualmente ha permitido entender que una investigación o un estudio sobre género y ciencia o sobre ciencia y feminismo no tiene por qué ser sobre las mujeres, sino sobre cómo, por ejemplo, ha afectado a la construcción de la ciencia que hemos heredado, lo que las mujeres y los hombres han sido y son "a partir de una dinámica compleja de fuerzas cognitivas, emocionales y sociales entretejidas" (Keller, 1991, 12).

\subsection{SOBRE FEMINISMO Y SOBRE CIENCIA}

Demasiadas veces se utiliza el término "feminismo" como si se tratase de un conjunto monolítico de creencias y de prácticas, cuando ni es un movimiento único en la actualidad, ni tampoco sus manifestaciones históricas han sido uniformes. Hay diferentes feminismos, y diferencias significativas entre quienes se denominan feministas, respecto de los análisis y de las prácticas más convenientes (Harding, 1996, 96) para hacer posible una sociedad en la que el sexo no sea una razón de desigualdad. Podemos hablar de feminismo del siglo de la Ilustración -con raíces en el siglo anterior-, momento en el que nació ya en grupos de mujeres una conciencia clara del papel que se les estaba asignando en el diseño de la nueva sociedad que entonces se 
proponía; feminismo del siglo XIX, que defendió las medidas que podían aplicarse a la situación de subordinación en la que vivían las mujeres -educación, sufragismo, trabajo, etc.-, pero sin lograr captar suficientemente todos los mecanismos que generaban y mantenían aquellos desequilibrios en las relaciones sociales en función del sexo; feminismos del siglo $\mathrm{XX}$-el liberal, el marxista, el radical, el que recurre a diversas escuelas psicoanalíticas, el académico- que, con su variedad de posturas ideológicas y de estrategias prácticas, no han podido neutralizar del todo la adaptabilidad histórica y cultural del modelo construido desde el patriarcado, no sólo en relación con la jerarquía cultural de sexo, sino también con la de clase social y la de raza.

Cuando la "mujer", objeto ideal, abstracto, estable, uniforme, convertido en sujeto del saber, se ha deconstruido en "mujeres" reales, concretas o anónimas, de diferentes épocas y sociedades", en un objeto "necesariamente plural, multiforme, pluridisciplinar" (Perrot, 1995, 41), y cuando se ha reconocido que el género carece de referentes fijos, es decir, que es un concepto histórico, ningún feminismo, y menos un sólo feminismo, puede representar y ofrecer una teoría que refleje la voz de una hablante naturalizada o esencializada que, en su mismo planteamiento, parte de que no existe.

Por otro lado, aunque la ciencia ha presentado hasta hace poco sus concepciones de la naturaleza y de la sociedad como reflexiones con vocación de ser objetivas y universalmente válidas - por haber sido producto de la razón, de la mente, y no del sentimiento, que implica subjetividad-, la hermenéutica de la sospecha y quienes cultivan cada una de las ciencias y su historia están indicando que estas concepciones han cambiado en el curso del tiempo bajo la influencia de factores condicionantes y responsables de que unas teorías y conclusiones adquirieran legitimidad social y otras no, y no únicamente por la lógica interna que generaban. Y la sociología del conocimiento ha incluido los estudios de ciencia y género entre sus intereses de atención, no sólo porque "investigan una profunda anomalía social en la práctica científica (la escasa presencia de la mujer)", sino especialmente porque "ponen en cuestión valores epistémicos como la neutralidad y la objetividad" (Echevarría, 1995, 29).

El que sigamos entendiendo y deseando el saber, prioritariamente, como "pretensión de verdad" (Ricoeur, 1987, 371) y la ciencia como conocimiento adquirido mediante la aplicación de un método científico que es verificable, que puede ser articulado en teorías y que es susceptible de ser comunicado con exactitud, no significa dejar de reconocer el hecho de que la ciencia haya

\footnotetext{
${ }^{3} \mathrm{El}$ paso del singular al plural no supone un mero cambio de número, sino un cambio de sentido. La realidad empíricamente observable de las cosas y de las personas es siempre plural. Cuando las formulamos en singular pasamos de lo concreto a lo abstracto y universal (Ramírez,
1992,21 ).
} 
sido, y siga siendo, una actividad y un producto personal y social que esté ampliando sus posibilidades de provisionalidad. En las puertas de un nuevo milenio, es ya más fácil afirmar que las creencias, incluso las científicas y los núcleos cognitivos de éstas, y más el sistema de creencias sobre el significado de lo masculino y de lo femenino, no son inmunes a las influencias culturales y a los intereses de personas y de grupos.

En este marco, encontrar explicaciones más próximas a la verdad implica desafiar conceptualizaciones muy importantes del conocimiento acumulado por la historia, e intentar redefinir tales conceptos, crear otros nuevos y buscar síntesis renovadas. Algo que se está viendo necesario en muchas ciencias, pero muy especialmente en las ciencias sociales (Abellán, 1991, 47-63).

\subsubsection{La necesaria redefinición de conceptos}

En el cuestionamiento del carácter epistemológico que se produjo en los estudios de la mujer -lo que igualmente se puso de manifiesto en otros ámbitos científicos-, se empezó dudando del principio de objetividad y de neutralidad del sujeto que conoce como algo incuestionable y se pasó a afirmar la existencia y el valor de lo subjetivo, la importancia del contexto y la posibilidad de conocimiento a partir de la experiencia directa, también desde la de las mujeres. Quienes se iniciaban en los estudios de la mujer los planteaban desde el convencimiento de que científicamente era necesario incorporar una perspectiva, una visión, un modo de ver, que no podía seguir siendo obviado; una voz que era igual de legítima, porque expresaba el punto de vista de las mujeres, de una mitad de la humanidad; de ahí su inicial insistencia en llamar la atención sobre la invisibilidad y la ausencia de las mujeres en todas las ciencias.

La desconfianza no era hacia la objetividad o hacia la vigilancia sobre el pensamiento ejercido por la epistemología, en lo que esas condiciones buscaban representar, sino hacia la forma concreta de objetividad y hacia el uso de la epistemología que subyacía en "el discurso filosófico de la modernidad" (Habermas, 1989), en la ciencia de la Ilustración. La ausencia de las mujeres no planteó entonces ningún problema serio ni en la normativa de la teoría democrática, ni en los presupuestos de los análisis científicos entonces formulados. Se planteaba como un problema no de la ciencia en cuanto tal, sino de una ciencia que asumía que los problemas que afectaban a los hombres eran los de toda la población y los del mundo -pues en ningún caso vislumbraron que esa visión estaba deformada por sus necesidades y deseos de género-, y que no daban cabida a la explicación de muchas de las cosas que resultaban problemáticas, o relevantes, para las mujeres. Unas posturas que hay que circunscribir a los límites de lo que ya muchos grupos entienden hoy como mala ciencia, sin extenderlas a toda la ciencia.

La reflexión sobre un modelo de racionalidad que incluya lo femenino puede parecer un lujo de un grupo de personas, aunque sea amplio, sólo 
preocupado por focalizar aspectos y dimensiones que fueron silenciadas por creer que carecían de relevancia en el diseño social y científico. Pero, observado con mayor detenimiento, es un proyecto con consecuencias potenciales que no se pueden desestimar, ya que contribuye positivamente a una modalidad de búsqueda del saber que ofrece mayores posibilidades de ampliar la base de quienes tienen el derecho a sentir los beneficios que él reporta.

De hecho, en los últimos años, ya se ha iniciado la formación de un corpus teórico que está contribuyendo a que algo cambie en todos los campos del conocimiento, al replantearse conceptos como los de racionalidad y de objetividad, y al pasar de la no consideración de la experiencia de la mitad de la población a la aceptación de que también forma parte de la historia. Y al hacerlo, se han empezado a descubrir los mecanismos económicos, políticos y psicológicos que mantienen sesgos en la ciencia, el sexismo entre ellos, y que debemos eliminar para que la naturaleza, los usos y las valoraciones en la búsqueda del saber sean más integradoras de todo lo humano. Porque hoy somos más conscientes de que tampoco es suficiente que las ciencias se ajusten de manera rigurosa a sus propias directrices de investigación objetiva, si lo hacen con independencia respecto de los valores y de los sentimientos.

Hay un mundo que no es el de la racionalidad, cada vez menos oculto para una parte de la ciencia: el de las emociones, de los sentimientos, del inconsciente individual y colectivo, de las particularidades sociales e históricas, de la experiencia -una realidad problemática para la ciencia-, y parte del proyecto de los Estudios de la Mujer consiste en poner de manifiesto la relación entre estos dos mundos, el demostrar cómo cada uno configura y forma al otro recíprocamente.

El comportamiento, el tiempo y el espacio, las percepciones, la experiencia vital de las mujeres, así como su visión e interpretación de la realidad han sido excluidos e ignorados en los corpus de conocimiento elaborados por los científicos centrados en la exclusiva experiencia masculina. El conocimiento legitimado a lo largo del tiempo se ha descubierto como androcéntrico, cuando sabemos que lo humano no es la proyección y generalización de lo masculino, porque también está sexuado en femenino. Entramos aquí en la cuestión de "los genéricos", que viene ocupando la reflexión de algunas filósofas desde hace aproximadamente dos décadas, en el intento de saber su génesis y su desarrollo posterior, es decir, sobre cómo se han elaborado, cómo se utilizan y qué legitimidad poseen (Amorós, 1987; Valcárcel, 1989).

Pero puede surgir la pregunta de si la crítica feminista del "objetivismo" tive objetividad igual a neutralidad axiológica- obliga al subjetivismo, al relativismo, si desemboca en el reemplazar unas lealtades de género por otras. Entiendo que lo que se quiere, más bien, es llegar a hipótesis que no dependan indefectiblemente de esas lealtades, dejando espacio para objetivos de búsqueda del saber que permita "elaborar teorías que representen con precisión las actividades de las mujeres como actividades sociales, y las relaciones 
sociales entre los géneros como un componente real de la historia humana" (Harding, 1996, 121).

\subsubsection{La teoría del género}

En las ciencias sociales, y más especialmente en la psicología y en la antropología, se había apuntado ya hace más de un siglo al hecho de que las conductas y roles atribuidos a hombres y a mujeres eran una construcción social y que no tenían que ver necesariamente con su realidad biológica (Stuart Mill, 1892), pero, hasta hace aproximadamente tres décadas, no se empezaron a aislar con mayor rigor lo que eran vías sociales pretrazadas de lo que eran manifestaciones derivadas de la naturaleza biológica de cada persona. Es en los años setenta cuando se acertó a acuñar el concepto de género que ha permitido explicar que hay comportamientos de hombres y comportamientos de mujeres que han sido construidos socialmente, y a los que no hemos de ver como determinados ni condicionados por el sexo al que la persona pertenece (Rivera, 1994, 157).

La insistencia en las connotaciones socioculturales del género, para distinguirlo de las connotaciones biológicas del concepto sexo, fue desvelando una asimetría "lógica" en el contenido y la valoración de la masculinidad y de la feminidad, porque asignaba metáforas dualistas -simbolismo de género-, dividía dicotómicamente las actividades sociales -estructura de género- y determinaba una forma de identidad individual -género individual(Harding, 1996, 17). Por lo tanto, teorizar la cuestión de la diferencia sexual, es decir, una teorización adecuada del género nos llevaría siempre a plantearnos cuestiones sobre las interacciones entre el simbolismo de género, el modo concreto de división social del trabajo o la actividad según el género y lo que constituye las identidades y deseos generizados en una cultura particular. Cuestiones que son pertinentes no sólo con respecto a la cultura de la ciencia entre los siglos XV y XVII en Europa, sino también respecto de las culturas que han apoyado la ciencia en los siglos posteriores (Harding, 1996, 50).

El género es una categoría fundamental en cuyo ámbito se asigna significado y valor a todas las realidades, además de ser una forma de organizar las relaciones sociales; la más antigua, universal y poderosa: se ha asignado género a los huracanes y a las montañas, a los barcos y a las naciones, y ha poblado nuestros sistemas de creencias, las instituciones, la arquitectura o la planificación urbana; ha reflejado, deformándolas, las divisiones del trabajo en una cultura, o las identidades de sexo y de género de sus partícipes. Todas las personas, también los hombres, han tenido una socialización de género. El lugar tanto de las mujeres como de los hombres en el sistema sexo-género está socialmente construido; y así lo avalan los estudios históricos, biológicos, antropológicos y psicológicos.

Lo que ha llevado al establecimiento de jerarquías y de discriminación unidas al género, pues, además de dividir la realidad en dos, masculino y 
femenino, uno de ellos es considerado de valor superior y goza de mayor prestigio, con lo que tal valoración puede suponer para quienes sufren en su proceso educativo esas formas de socialización. El tener que asimilar información de forma dicotomizada, en la que los niños encuentran una mayor facilidad para identificarse con un modelo que se presenta como superior, al mismo tiempo que las niñas perciben la falta de valoración pública de lo que han de integrar en su propia personalidad, se puede convertir demasiadas veces en fuente de contradicciones (Acker, 1995, 115-116).

Lo que solemos considerar problemas, conceptos, teorías y verdades trascendentales que abarcan todo lo humano son, en muchos casos, productos del pensamiento de quienes los crean, marcados por imágenes y estereotipos sexuales sobre las identidades, las conductas, los roles y los deseos. Gran parte de lo que los hombres consideran "naturaleza" en oposición a "cultura", para las mujeres pertenece a la "cultura". El segundo sexo, de Simone de Beauvoir, despertó en 1949 la conciencia de la construcción social de las diferencias sexuales percibidas -"no se nace siendo mujer, se llega a serlo" (Beauvoir, 1987, 13)-, y la bibliografía posterior lo ha confirmado. Es una experiencia y una convicción que otra mujer, Luce Irigaray, sentiría veinte años más tarde, ya en el marco de un nuevo sentido de lo femenino, al decir que "nosotras todavía no hemos nacido mujeres" (Irigaray, 1987, 78). El destino de la población femenina no está en su biología; sus destrezas domésticas, asistenciales y afectivas tienen una génesis social, no natural, de ahí que los movimientos de mujeres se opongan a la división de las actividades mentales, manuales y asistenciales en razón del sexo. Aunque, al mismo tiempo, quieren afirmar y defender que en la sociedad actual no deben verse obligadas a negar que son mujeres para poder sobrevivir.

La construcción social de las diferencias que crean desigualdad es explicada por teorías como el determinismo biológico, el constructivismo y la interacción entre biología y cultura. Los resultados de numerosas investigaciones en estos campos han puesto de manifiesto la diversidad de contenidos de lo femenino y de lo masculino que están presentes en distintas culturas y épocas. Han señalado que las actividades y formas culturales asignadas a cada sexo, así como su significación, diferían mucho entre sociedades que se han conocido en fases diferentes de desarrollo, e incluso en una misma sociedad a través del tiempo; datos que ayudan a poder afirmar que eso que llamamos género varía de una sociedad a otra ${ }^{4}$ y que las diferencias de género se deben más a factores culturales que a características biológicas, lo que hace posible que haya más de dos expresiones culturales del género.

El potencial analítico del concepto género permitió mostrar estos factores culturales que uniformaban, pero que igualmente pueden diversificar, y el

\footnotetext{
${ }^{4}$ Las investigaciones de Margaret MEAD (1935), por ejemplo, constituyeron una temprana e
importante contribución.
} 
del sistema de género (Rubin, 1975) hizo posible visibilizar cómo se organizaban las sociedades para crear, para mantener y para reproducir esas características y esos comportamientos, a los que no ajustarse implicaba sanción social. Como en otras áreas de investigación psicosocial, se separó la biología de la cultura y adquirió visibilidad propia lo que hasta entonces había permanecido oculto bajo el concepto de esencialidad.

La utilización de este concepto género por parte de las teóricas feministas ayudó al reconocimiento de la legitimidad académica de los Estudios de la Mujer, pues se percibía como una formulación más cercana y objetiva de los problemas que quería analizar y con mayores posibilidades de seriedad y de rigor. Y el de sistema de género ayudó a entender que dichos estudios no podían quedar reducidos al sexo, como sinónimo de sexualidad y de reproducción biológica, sino que tenían que abarcar todos aquellos ámbitos desde los que la sociedad y las relaciones que en ella se producen pueden ser conocidas. Acercamientos que han permitido percibir mejor la pluralidad de formas con que hoy las mujeres están presentes en el mundo y en la historia (Flecha, 1996).

La introducción en los últimos años de esta perspectiva en diferentes campos de investigación ha pretendido responder a tres intereses fundamentales:

Primero: a la búsqueda de un modo que permita romper con las representaciones tradicionales, procedentes del esencialismo o del biologicismo, de las relaciones sociales que se establecen entre hombres y mujeres.

Segundo: a la necesidad de conocer mejor cómo se producen las relaciones de poder entre hombres y mujeres.

Tercero: a la pertinencia de ajustar la teoría feminista a ciertos cánones de la legitimidad académica, a lo que parece ha contribuido la sustitución del término mujer por el de género (Irigaray, 1987, 78).

Y entre los resultados hay uno cada vez más evidente, el que demuestra que hablar de género femenino representa una abreviación del pensamiento, si no indicamos las mujeres concretas que a él pertenecen (Zamboni, 1996, 33). Puede haber muchas manifestaciones culturales del género, por lo que esta categoría de análisis no se puede convertir en un nuevo concepto tan esencialista y homogeneizador como fue durante siglos el de "mujer" (Young, 1997, 100).

\subsection{PROPUESTAS DE UNA EPISTEMOLOGÍA DESDE LAS MUJERES}

Esta perspectiva tiene por objeto, consecuentemente, analizar algunos de los presupuestos metodológicos y de las prácticas científicas que han contri- 
buido a crear o a reproducir diferencias sociales en razón del sexo a lo largo de la historia y en la actualidad, provocando la desigualdad entre personas. Se propone buscar procedimientos que eviten la peligrosa ficción de lo exclusivamente humano -entendido unas veces con sentido universal y otras sólo masculino-, naturalizado y esencializado, así como la deformación y desajustes derivados de esa ficción.

Con este sentido, una epistemología encaminada a acceder a unos saberes que incluyan también las dimensiones señaladas, tendrá que atender:

- A cómo los conceptos de la persona que investiga, del mundo objeto de conocimiento y de los procesos que lo hacen posible reflejan la unidad de las actividades manuales, mentales y emocionales, desestimando los dualismos cartesianos de intelecto frente a cuerpo y frente a sentimiento y emoción; en qué medida toma en consideración la legitimidad de las apelaciones a la subjetividad y, por lo tanto, a la necesidad de unir los campos intelectual y emocional; cómo sustituye el predominio del reduccionismo y de la linealidad por la atención al holismo y a la complejidad de la persona.

Un modo de conocer que busca trascender y oponerse a las dicotomías entre trabajo teórico y compromiso con la práctica y a las que se aceptan entre un concepto de feminidad relacional frente a otro de masculinidad abstracta. En este modo de conocer se tiene en cuenta la subjetividad, la experiencia y las diferencias interpersonales (Lennon, 1995, 133-143).

- A una teoría que postula el retorno de las mujeres para lograr un conocimiento más adecuado del mundo y de la historia de la humanidad en él; que se propone desvelar las raíces sociales de unos saberes abstractos y aparentemente universales pero que, en realidad, han sido el fruto de la experiencia y de la reflexión de unos filósofos y científicos que los han interpretado como paradigma de la experiencia humana; camino de conocimiento en el que la experiencia femenina estaba destinada a desaparecer. Una buena teoría tiene que considerar a las mujeres y a su experiencia como hechos evidentes de por sí, origen de identidad y de acción colectivas (Scott, 19962, 85), como un elemento imprescindible en la construcción de una buena ciencia.

Pensar desde la perspectiva de las mujeres -desde mujeres concretas y diferentes- puede iluminar algunos aspectos de la totalidad social que habían sido suprimidos por la visión predominante. Quizás este punto de vista no se libre tampoco de una cierta parcialidad, pero los resultados que produzca harán que el conjunto sea más verdadero que el anterior:

- A una descripción histórica, a reconstruir la génesis, porque ello permite mostrar mejor los cambios que se han producido en las formas de comprensión y en la vida social en la que éstas surgían. El papel de la 
historia en la ciencia es el de descifrar las condiciones previas de su propia producción, en donde precisamente pueden encontrarse diversos sesgos y prejuicios que se introducen en las investigaciones, sobre todo en la fase de identificación y de definición de los problemas, oscureciendo la observación de la realidad y el conocimiento de la misma.

- A poner en duda el supuesto de que la identidad social de quien observa es irrelevante, a cuestionar la capacidad de las normas metodológicas para eliminar por sí mismas los sesgos androcéntricos. Quien investiga interviene en la selección, focalización, sentido, significado, etc., de las fuentes y datos con las que se encuentra. Establece una relación con el objeto de estudio que afecta a la operación que está realizando; reorganiza creadoramente e interpreta las huellas y los vestigios con los que está trabajando, algo que ya hace más de treinta años puso de manifiesto el historiador Edward Carr $\left(1981^{10}\right)$. El pasado, las experiencias, la memoria, las expectativas, el tiempo y el lugar desde el que la investigación se realiza -un tiempo y un lugar social, cultural, científico y personal- abren y cierran perspectivas y posibilidades.

- A proponer estrategias "del punto de vista" que "se basan en las características compartidas por las mujeres como grupo social y los hombres como grupo social" (Harding, 1996, 141); modos de conocer que sostienen que la conceptualización de la experiencia social de las mujeres puede aportar las bases para una descripción y para una comprensión menos deformada no sólo de las mujeres, sino también de los hombres y del mundo que nos rodea. Pues no sólo se reclama el derecho a nombrar, a definir y a explicar el mundo desde la experiencia femenina, sino también a expresar con ello que la sociedad está compuesta por hombres y por mujeres.

\subsubsection{Cambiar el mundo, no el pasado}

Los primeros objetivos en esta forma de hacer ciencia se centraron casi exclusivamente en suplir la ausencia de mujeres en la ciencia y en descubrir las circunstancias que habían causado esa ausencia. En el marco de las prácticas políticas de la igualdad, la contribución de las investigadoras era ofrecer datos de suplencia. Pero al desarrollar los proyectos que conducían a esa finalidad, se vislumbró algo considerado como más importante, el hecho de las consecuencias científicas que tenía la escasa representación de las mujeres en la ciencia (Keller, 1991, 189).

Este hallazgo llevó a desviar la atención "desde la cuestión de la mujer en la ciencia, a la cuestión, más radical, de la ciencia en el feminismo" (Harding, $1996,27)$. Una postura que permitió empezar a examinar los efectos de las relaciones sociales entre los géneros sobre las ideas y sobre las prácticas científicas, culturales, sociales, con el convencimiento cada vez más nítido de que "la incorporación de la mujer al proceso de producción de la ciencia 
figura entre las condiciones necesarias, pero no suficientes, para la incorporación de la ciencia al proceso de liberación de la mujer" (Durán, 1982, 3031). El problema a resolver era, por lo tanto, cómo pueden recibir las mujeres un trato más equitativo dentro de la ciencia y como podría utilizarse la ciencia con fines emancipadores para las mujeres. La finalidad prioritaria no era cambiar el pasado de las generaciones que nos precedieron, sino cambiar el mundo en el que ahora vivimos y preparar el futuro que está por venir.

Pasar, por lo tanto, desde una postura reformista, que abría la posibilidad de mejorar la ciencia, al esfuerzo para una transformación de les fundamentos conceptuales y de las premisas epistemológicas de la misma. Modo de hacer que se sitúa en una concepción del saber que se entiende como una actividad plenamente social en la que han de reflejarse, consciente e inevitablemente, los compromisos de quienes investigan; que selecciona los problemas, los conceptos, las teorías, las metodologías, las interpretaciones y los usos para el desarrollo de las estructuras intelectuales y sociales de la ciencia, teniendo presentes objetivos morales y políticos, y no sólo cognitivos. En esta línea, el punto de vista feminista reconoce que se encamina "hacia una posición social interesada", en lo que ésta significa de compromiso más que de sesgo (Harding, 1996, 129).

Algo, sin duda, está transformando las relaciones sociales entre razas, clases y culturas, lo mismo que entre los sexos, a un paso mucho más rápido que nuestras posibilidades de teorización. De ahí que las temáticas que constituyen cada objeto de estudio estén sometidas a constantes y profundas transformaciones.

\subsubsection{Tareas todavia pendientes}

La incorporación de estas perspectivas en las últimas décadas ha ayudado a flexibilizar certezas, a debilitar límites intradisciplinares e interdisciplinares, a enriquecer el debate intelectual de muchas personas y grupos. Los Estudios de las Mujeres han abierto una vía de análisis que está permitiendo desde hace años (Durán, 1982a, 30-32; Durán, 1982c, 14-15), pero que no está concluido, abordar el conocimiento con nuevas y enriquecedoras posibilidades. Posibilidades que, en parte, se han aprovechado ya y que, en parte, se presentan como tareas aún pendientes. Me referiré a algunas para terminar:

1. La conveniencia de una mirada crítica a las ciencias y a las disciplinas académicas tradicionales; por ejemplo, a una historia que olvida a las mujeres o a una economía que no cuenta con el trabajo doméstico, lo mismo que al divorcio entre ellas, que impide el acercamiento interdisciplinar a realidades que lo requieren, como, en este caso, la situación de las mujeres en el pasado y en el presente.

2. La necesidad de una nueva profundización de los conceptos y de los supuestos fundamentales de las ciencias y de las humanidades, que 
pueden seguir incorporando sesgos encubiertos, tanto en su teoría como en los procedimientos de observación y de recogida de datos. Dicotomías, por ejemplo, que más que explicar, esconden: natural/cultural, doméstico/público, reproducción/producción (Narotzky, 1995, 13 y 31 ).

3. El desarrollo de un cuerpo de conocimientos en cada una de las disciplinas que transmita una información más sistemática, e integrada en el conjunto, de las mujeres y de cómo se ha ido construyendo el género en las culturas del pasado y en las actuales.

4. Mejorar las disciplinas y campos de estudio mediante la incorporación de nuevos datos, métodos, teorías y marcos conceptuales que tengan también en cuenta a las mujeres como punto de referencia y medida de lo humano. Que se detenga en el análisis de la génesis y de la distribución de unos conocimientos que no son neutros, sino que remiten a situaciones sociales y culturales concretas, y que proponga pensarlos y reelaborarlos de nuevo.

5. Trabajar con un concepto de mujeres no esencialista ni etnocéntrico, sino que incluya también la consideración de la raza, de la procedencia social y geográfica, de la cultura y de la religión, como variables que afectan a las identidades individuales.

6. En definitiva, ir consiguiendo un saber más global y pluridisciplinar en el modo de ser transmitido y difundido -frente a una tendencia a la sectorialización de intereses y de tratamientos- que permita crear una base de conocimientos que reconozcan a las mujeres, y en los que puedan ser educadas las nuevas generaciones. 


\section{BIBLIOGRAFÍA}

ABELLÁN, J. (1991). "Historia de los conceptos (begriffsgeschichte) e historia social". En Castillo, S. (coord.). La Historia social en España. Madrid, Siglo XIX, 47-63.

ACKER, S. (1995). Género y educación. Reflexiones sociológicas sobre las mujeres, la enseñanza y el feminismo. Madrid, Narcea.

ADDIS SABA, M. (1992). "Women's Studies in Italy: the History of feminist historiography". Women's Studies Quartely, 20 (3-4).

AMORÓS, C. (1987). "Espacio de los iguales, espacio de los idénticos". ARBOR, CXXVIII, (503-504), 113127.

ANEF (1995). "Études féministes et études sur les femmes en France en 1995". En Ephesia. La place des femmes. Les enjeux de l'identite et de l'égalite au regard des sciences sociales. París, La Découverte, 689-710.

BALlaríN, P., GALLEGo, T. y MARTINEZ, I. (1995). Los estudios de las Mujeres en las Universidades españolas 1975-1991. Madrid, Instituto de la Mujer.

BEAUVOIR, S. de (1987). El segundo sexo. Buenos Aires, Siglo XXI, vol. 2.

BIRD, E. (1995). "Women Studies in the United Kingdom". En Sigma. Conference on Women's Studies in Europe: European Universities Networks, Coimbra, Comisión Europea, 1995, 31 pp.

CARR, E. $\left(1981^{10}\right)$. ¿Qué es la historia? Barcelona, Seix Barral.

COMISIÓN EUROPEA (1992): Grace: Les etudes feministes dans la Communauté Europeenne. Bruxelas,, Comisión Europea, 4 vols.

CHÁTHAIGH, D. (1995). "Women's Studies in Ireland". En Sigma. Conference on Women's Studies in Europe (CWSE): European Universities Networks. Coimbra, Comisión Europea, $18 \mathrm{pp}$.
DEGLER, C. (1975). Is There a History of women?. Oxford, Clarendon Press.

DONNE D'EUROPA (1984). Donne $e$ ricerca nei dieci paesi della Comunitá Europea. Commissione della Comunitá Europea, suppl. n1 18, 55 pp.

DURAN HERAS, A. (1982a). Liberación y utopía: la mujer ante la ciencia. Madrid, Akal Editor.

- (1982b). "Notas para una ruptura paradigmática”. En Gallego, T. (Ed.): Nuevas perspectivas sobre la mujer. Madrid, Universidad Autónoma, Tomo II, 13-16.

ECHEVARRÍA, J. (1995). Filosofía de la ciencia. Madrid, Akal.

EQUIPE SIMONE (1996). Grace: Rapport a l'unite pour l'egalite des chances. Universite Toulouse-Le Mirail.

ERICSSON, E. (1995). "Women's Studies National Report Sweden”. En Sigma (CWSE): European Universities Networks. Coimbra, Comisión Europea, $41 \mathrm{pp}$.

FLECHA GARCÍA, C. (1987). "La mujer en la enseñanza universitaria". En VV.AA.: La Universidad hoy en un contexto internacional. Málaga, Publicaciones Universidad, pp. 582-591.

- (1996): "Las mujeres, del género a la diferencia". En Documentación Social, 105, pp. 73-92.

GALINO CARRILLO, A. (1972). "La mujer en la educación y en la cultura". En VV.AA.: Congreso Internacional de la Mujer. Madrid, Almena, pp. 109-127.

GOMEZ FERRER, G. y otras (1995). Las relaciones de género, Madrid, Marcial Pons.

HABERMAS, J. (1989). El discurso filosófico de la modernidad. Madrid, Tecnos.

HANMER, J. et al. (1994). Women's Studies and European Integration. Report Equal Opportunities Unit of the European Commi, Bruxelles. 
HARDING, S. (1996). Ciencia y feminismo. Madrid, Morata.

INSTITUTO DE LA MUJER (1988). La presencia de las mujeres en el sistema educativo. Madrid, Instituto de la Mujer.

INSTITUTO NACIONAL DE ESTADÍSTICA (1991 a 1995). Estadística de la Enseñanza Superior en España. Madrid, INE.

IRIGARAY, L. (1987). Sexes et parentés. París, Éditions Minuit.

JANSEN, W.-ALST, J. (1995). "Women's Studies in the Netherlands". En Sigma (CWSE): European Universities Networks, Coimbra, Comisión Europea, $43 \mathrm{pp}$.

KELLER, E. F. (1991). Reflexiones sobre género y ciencia. Valencia, Alfons el Magnànim.

KROPS, C. (ed.) (1997). European Women's Studies Guide, Utrecht, Wise.

LENNON, K. (1995). "Gender and Knowledge". Journal of Gender Studies, 4, (2), 133-143.

MARTIN, J. "Recherches et études fèministes en France: une synthèse des processus institutionnalisants des enseignements et de la recherche entre 1970 et 1990". Ressources for Feminist Research, 23, (1-2), pp. 2428.

MEAD, M. (1935). Sex and Temperament in Three Primitive Societies. New York, New American Library. (Sexo y Temperamento. Barcelona, Altaya, 1994).

MORENO, A. (1977). El movimiento feminista en España. Barcelona, Anagrama.

MÜLLER, U. (1995). “Women's and gender studies in Germany". En Sigma (CWSE): European Universities Networks. Coimbra, Comisión Europea, 29 pp.

NAROTZKY, S. (1995). Mujer, mujeres, género: una aproximación crítica al estudio de las mujeres en las Ciencias Sociales. Madrid, C.S.I.C.

NASH, M. (1988). "Conceptualización y desarrollo de los estudios en torno a las mujeres: un panorama internacional". Papers: Revista de Sociología, 30, pp. 13-22.

PARLAMENTO EUROPEO (1994). Informe de la comisión de derechos de la mujer sobre el personal cientifico dedicado a la $I+D$ en Europa. Parlamento Europeo, $13 \mathrm{pp}$.

PERROT, M. (Dir.) (1984). Une histoire des femmes est-elle possible? MarsellaParís, Ed. Rivages.

- (1995). “Identité, Égalité, Différence. Le regard de l'Histoire". En Ephesia: La place des femmes: Les enjeux de l'identite et de l'egalite au regard des sciences sociales, Paris, La Découverte, 39-56.

RAMÍREZ, J. L. (1992). "El significado del silencio y el silencio del significado". En Castilla del Pino, C. (Ed.): El silencio. Madrid, Alianza, 19-32.

REYNOLDS SOUSA, I. (1993). "Estudos sobre as mulheres en Portugal". Cadernos Condiçaa Feminina, 38.

RICOEUR, P. (19903). Historia y verdad. Madrid, Encuentro.

RIVERA GARRETAS, M. ${ }^{\mathrm{a}}$ M. (1994). Nombrar el mundo en femenino. Pensamiento de las mujeres y teoría feminista. Barcelona, Icaria.

- (1996). "La historia de las mujeres, ¿es la historia?". En VV. AA.: Aspectos didácticos de Geografía e Historia. Zaragoza, Publicaciones Universidad, 41-61.

RUBIN, G. (1975). "The Traffic in Women: Notes on the Political Economy of Sex". En Reiter, R. (Ed.): Toward an Anthropology of Women. Nueva York, Monthly Review Press, 157210.

SCOTT, J. (1996"). "Historia de las Mujeres". En Burke, P. (Ed.): Formas de hacer Historia. Madrid, Alianza, 59-88.

STUART MILL, J. (1892). La esclavitud femenina. Madrid, Compañía Impresores y Libreros.

TOBE, M.-STEEN (Eds.) (1993). European Women's Studies Guide. Utrecht, Women's International Studies Europe. 
VALCÁRCEL, A. (1989). "Las figuras de la heteronomía: del vosotros al yo". En Maquieira, V.-Sánchez, C. (Ed.). Mujeres y hombres en la formación del pensamiento occidental. Madrid, Universidad Autónoma, vol. I, 87108.
YOUNG, S. (1997). Changing the Wor(l)d. Discourse, Politics, and the Feminist Movement. New York-London, Routledge.

ZAMBONI, Ch. (1996), "Lo inaudito". En Diótima. Traer al mundo el mundo. Barcelona, Icaria, 23-39.

\section{RESUMEN}

En este artículo se aborda el proceso de desarrollo y la actualidad de los Estudios de la Mujer en las Universidades de muchos países, incluida España, como consecuencia de la incorporación de nuevas propuestas epistemológicas a numerosos ámbitos científicos. Se analiza el concepto de género y también la necesidad que está provocando el diálogo y la confrontación entre género y ciencia, de redefinir y/o de transformar muchos de los supuestos fundamentales que han prevalecido en las prácticas cientificas. Se pone de manifiesto que, en el marco más amplio de los estudios sociales de la ciencia, la reflexión que están realizando en las últimas décadas especialmente muchas mujeres, desde diversos modos de entender los análisis y las prácticas feministas, ha contribuido a centrar la atención en algunas propuestas epistemológicas que eviten la reproducción de desigualdades en función del sexo. Y que el análisis crítico de las circunstancias que han causado la ausencia de mujeres en la ciencia y las consecuencias científicas de ello no pretende rectificar el pasado, sino abrir caminos a un conocimiento no sesgado por planteamientos androcéntricos.

Se reconoce que la incorporación de esta perspectiva ha ayudado a flexibilizar certezas, a debilitar límites intradisciplinares y a enriquecer el debate intelectual de muchas personas y grupos.

Palabras clave: Estudios de la Mujer. Universidad. Ciencia. Género. Feminismo. Mujeres. Siglo XX. España. Europa. Estados Unidos. 


\section{ABSTRACT}

This paper deals with the process of development and the present of the Women's Studies at the universities of many countries, Spain included, as a consequence of the incorporation of new epistemological proposals to many scientific realms. The concept of gender is analized as well as the need, aroused by the dialog and confrontation between gender and science, of redefining and/or transforming many of those fundamental assumptions which have prevailed in scientific practices. It is shown that within the wider frame of the social studies of science, the reflection accomplished during the last decades, specially by many women, from different ways of understanding feminist analysis and practices, has contributed to focus attention upon some epistemological proposals that avoid the reproduction of differences because of sex. And also that the critical analysis of the circumstances which have caused the absence of women in science and the scientific consequences of it, does not attempt to straighten out the past, but to open ways to a knowledge not biased by androcentric positions.

It is acknowledged that the incorporation of this perspective has helped to make certainties more flexible, weaken interdisciplinar limits, and enrich the intellectual debate of many people and groups.

Key words: Women's Studies. University. Science. Gender. Feminism. Women. XXth Century. Spain. Europe. United States. 